\title{
Passively versus Actively Detected Malaria: Similar Genetic Diversity but Different Complexity of Infection
}

\author{
Zuleima Pava, ${ }^{1}$ Irene Handayuni, ${ }^{1}$ Leily Trianty, ${ }^{2}$ Retno A. S. Utami, ${ }^{2}$ Yusrifar K. Tirta, ${ }^{2}$ Agatha M. Puspitasari, ${ }^{2}$ \\ Faustina Burdam, ${ }^{3,4,5}$ Enny Kenangalem, ${ }^{3,4}$ Grennady Wirjanata, ${ }^{1}$ Steven Kho, ${ }^{1}$ Hidayat Trimarsanto, ${ }^{2}$ Nicholas Anstey, ${ }^{1}$ \\ Jeanne Rini Poespoprodjo, ${ }^{3,4,5}$ Rintis Noviyanti, ${ }^{2}$ Ric N. Price, ${ }^{1,6}$ Jutta Marfurt, ${ }^{1} \dagger$ and Sarah Auburn ${ }^{1 *} \dagger$ \\ ${ }^{1}$ Global and Tropical Health Division, Menzies School of Health Research, Charles Darwin University, Darwin, Northern Territory, Australia; ${ }^{2}$ Eijkman \\ Institute for Molecular Biology, Jakarta, Indonesia; ${ }^{3}$ Mimika District Health Authority, Timika, Papua, Indonesia; ${ }^{4}$ Timika Malaria Research \\ Programme, Papuan Health and Community Development Foundation, Timika, Papua, Indonesia; ${ }^{5}$ Maternal and Child Health and \\ Reproductive Health, Department of Public Health, Faculty of Medicine, Universitas Gadjah Mada, Yogyakarta, Indonesia; ${ }^{6}$ Centre for Tropical \\ Medicine and Global Health, Nuffield Department of Clinical Medicine, University of Oxford, Oxford, United Kingdom
}

\begin{abstract}
The surveillance of malaria is generally undertaken on the assumption that samples passively collected at health facilities are comparable to or representative of the broader Plasmodium reservoir circulating in the community. Further characterization and comparability of the hidden asymptomatic parasite reservoir are needed to inform on the potential impact of sampling bias. This study explores the impact of sampling strategy on molecular surveillance by comparing the genetic make-up of Plasmodium falciparum and Plasmodium vivax isolates collected by passive versus active case detection. Sympatric isolates of $P$. falciparum and $P$. vivax were collected from a large community survey and ongoing clinical surveillance studies undertaken in the hypomesoendemic setting of Mimika District (Papua, Indonesia). Plasmodium falciparum isolates were genotyped at nine microsatellite loci and $P$. vivax at eight loci. Measures of diversity and differentiation were used to compare different patient and parasitological sample groups. The results demonstrated that passively detected cases (symptomatic) had comparable population diversity to those circulating in the community (asymptomatic) in both species. In addition, asymptomatic patent infections were as diverse as subpatent infections. However, a significant difference in multiplicity of infection (MOI) and percentage of polyclonal infections was observed between actively and passively detected $P$. vivax cases (mean MOI: $1.7 \pm 0.7$ versus $1.4 \pm 1.4$, respectively; $P=0.001$ ). The study findings infer that, in hypomesoendemic settings, passive sampling is appropriate for molecular parasite surveillance strategies using the predominant clone in any given infection; however, the findings suggest caution when analyzing complexity of infection. Further evaluation is required in other endemic settings.
\end{abstract}

\section{BACKGROUND}

Calls for the global elimination of malaria have renewed political interest in public health interventions, reigniting control activities and placing elimination back on the agenda for many endemic countries. ${ }^{1}$ With the wide-scale deployment of key intervention strategies such as early diagnosis, prompt treatment, and intense vector control, 33 countries have reached the malaria elimination stage between 2000 and 2015 . $^{2}$ However, as the incidence of malaria falls in endemic settings, increasingly sensitive diagnostic tools are revealing large reservoirs of low-density malaria infections highlighting a far greater 'depth' of malaria burden. ${ }^{3-6}$ These subpatent infections are not routinely detectable by conventional microscopy and can persist for several months without clinical manifestations. Both of the predominant species, $P$. falciparum and $P$. vivax, are able to cause submicroscopic infections, although the relative proportion of subpatent cases tends to be higher in $P$. vivax than $P$. falciparum. ${ }^{2,7}$

Subpatent infections can be detected in a wide range of malaria-endemic settings, with the proportion tending to rise in low transmission areas. ${ }^{7-9}$ As countries approach malaria elimination, it is crucial to identify every infection to stop ongoing transmission and prevent resurgence. In this context, intense surveillance is required to monitor clinical and parasitological changes that can inform the most effective intervention strategies.

\footnotetext{
*Address correspondence to Sarah Auburn, Menzies School of Health Research, PO Box 41096, Casuarina, Darwin, NT 0811, Australia. E-mail: sarah.auburn@menzies.edu.au

†These authors contributed equally to the work.
}

The majority of such surveillance efforts have been undertaken at health facilities, recruiting symptomatic individuals with patent parasitaemia seeking treatment ("passive case detection"). As malaria elimination efforts progress, more indepth knowledge of the parasite population in asymptomatic community cases is needed, as this reservoir contributes a greater proportion of the total parasite burden. An important question is whether the genetic make-up of actively detected parasites is adequately represented by those detected passively, or whether the different sampling strategies can result in a significant bias which can affect the interpretation of a given study. The surveillance of antimalarial drug resistance could be particularly vulnerable to sampling bias, because phenotypic and molecular studies are generally applied to clinical isolates with little focus given to the asymptomatic population. In the case of ex vivo drug susceptibility testing, stringent criteria are applied which can result in even more constrained sampling. ${ }^{10,11}$ Furthermore, in pre-elimination settings, the low number of malaria cases in parasite population genetic studies often requires pooling of samples from patients with different disease presentations, collected using different sampling strategies and methodologies. These studies are being used increasingly to inform local trends in parasite transmission as reflected by measures of within-host and population diversity. ${ }^{12,13} \mathrm{~A}$ recent population genetic study of $P$. falciparum in a low endemic area of Zambia inferred that actively and passively detected infections formed distinct subpopulations with differing levels of diversity, but the study was partly constrained by the difference in years of collection between the actively and passively detected cases. ${ }^{14}$ Another study conducted in the higher endemic setting of Mimika 
District, Papua, Indonesia, using samples from both clinical and community cases revealed a subpopulation of $P$. falciparum isolates only observed in community cases. ${ }^{15}$ However, the latter study did not directly investigate the level of genetic differentiation or differences in population diversity and infection complexity between the actively and passively detected $P$. falciparum isolates. Furthermore, to date, no studies have directly compared the genetic make-up between actively and passively detected $P$. vivax infections, which contribute a large burden of malaria infections in the AsiaPacific region.

This study used sympatric $P$. falciparum and $P$. vivax isolates obtained from a community survey and ongoing clinical surveillance studies in Mimika District, Papua, Indonesia, to compare the genetic make-up of samples collected by passive versus active case detection, and between patent and subpatent groups.

\section{MATERIALS AND METHODS}

Study site and sampling. Passive case detection. The study was performed in Mimika District, Papua, Indonesia. Nearly $90 \%$ of the population of approximately 182,000 people live in lowland areas where unstable malaria transmission occurs. Although all four species of human-only infecting Plasmodium parasites can be found in the area, $P$. falciparum and $P$. vivax are the most common species causing $41 \%$ and $49 \%$ of malaria monospecies infections, respectively. Plasmodium malariae accounts for approximately $4 \%$ of malaria cases and less than $1 \%$ of cases are caused by $P$. ovale. Mixed-species malaria infections can be found in up to $6 \%$ of cases. ${ }^{16}$ Rumah Sakit Mitra Masyarakat (RSMM) is the main hospital in the district. RSMM is located in the capital city, Timika, and serves approximately 150,000 people, assessing up to 300 patients/day, 6 days/week. Patients with uncomplicated malaria due to any Plasmodium species are treated with dihydroartemisinin-piperaquine as per the national first-line protocol. ${ }^{17}$

Samples at the RSMM were collected within the framework of two studies. Between March 2011 and August 2014, patients presenting with an uncomplicated $P$. vivax or $P$. falciparum monoinfection, a peripheral parasitaemia between $1,000 \mu \mathrm{L}^{-1}$ and $80,000 \mu \mathrm{L}^{-1}$, as determined by microscopic examination, and more than $70 \%$ of ring stage parasites were invited to participate in an ongoing ex vivo antimalarial susceptibility surveillance study (ex vivo susceptibility study, ESS). Between March 2015 and February 2016, patients presenting with uncomplicated $P$. falciparum or $P$. vivax malaria with peripheral parasitaemia greater than $1,000 \mu \mathrm{L}^{-1}$ and $250 \mu \mathrm{L}^{-1}$, respectively, but with no restriction by parasite stage, were invited to participate in an ongoing treatment efficacy study (TES). After obtaining written consent, $5 \mathrm{~mL}$ of venous blood was collected for all patients by venipuncture and filtered using cellulose columns to deplete the host white blood cells. ${ }^{18}$ The postfiltration packed infected red blood cells were used for DNA extraction.

Active case detection. Active case detection was undertaken within the framework of a cross-sectional survey conducted in Timika between April and July 2013. Details on the sampling methods of the survey can be found elsewhere. ${ }^{16}$ Briefly, 800 houses were selected by cluster randomized sampling. First, the four largest subdistricts were chosen purposively. Consecutively, the number of clusters per district was calculated according to their respective relative population. Finally, 20 houses were chosen randomly within each cluster, following World Health Organization recommendations. Individuals living in the same house and residing in the study area for at least 6 months were included in the study. Symptomatic patients were defined as any individual with a current fever episode (i.e., axillary temperature > $37.5^{\circ} \mathrm{C}$ ) or in the last 24 hours. Venous blood from one adult volunteer and $200 \mu \mathrm{L}$ of capillary blood in a Microtainer ${ }^{\mathrm{TM}}$ from the remaining members of each household were collected and used for blood film examination, hemoglobin measurement, and molecular analysis.

DNA extraction and species confirmation. Plasmodium species was assessed initially by microscopy using Giemsastained thick and thin blood films. Peripheral parasitaemia was determined from the number of parasites per 200 white blood cells, assuming a white cell count of 7,300 cells $/ \mu \mathrm{L}$. Asexual stage composition was determined by classifying 100 parasites into ring, trophozoite, or schizont stage on examination of a thick smear.

For actively detected cases, genomic DNA (gDNA) was extracted from $50 \mu \mathrm{L}$ packed red blood cell (RBC) pellets using the QIAamp 96 DNA Blood Kit (Qiagen, Chadstone, Victoria, Australia). For passively detected cases, $2 \mathrm{~mL}$ of packed RBCs were extracted using the QIAamp DNA Midi Kit (Qiagen, Chadstone, Victoria, Australia). Plasmodium species confirmation was undertaken in duplicate with $2 \mu \mathrm{L}$ gDNA template using a nested PCR protocol as described elsewhere. ${ }^{19}$ Plasmodium falciparum, $P$. vivax, $P$. malariae, and $P$. ovale small-subunit $r$ RNA DNA clones (MRA-177, MRA-178, MRA-179, and MRA-180; ATCC, Manassas, VA) were used as positive controls.

Microsatellite typing. Plasmodium falciparum genotyping was conducted using nine previously described short tandem repeat (STR) markers (ARAll, PfPK2, poly-alpha, TA1, TA42, TA60, TA81, TA87, and TA109), following the protocol described by Anderson et al. ${ }^{20}$ For $P$. vivax genotyping, nine previously described STR markers selected as a consensus panel by the Asia Pacific Malaria Elimination Network Vivax Working Group (Pv3.27, MS16, MS1, MS5, MS8, MS10, MS12, MS20, and msp1F3) were used following previously described protocols. ${ }^{21,22}$ The fluorescently labeled PCR products were separated by capillary electrophoresis on an ABI 3100 Genetic Analyser with GeneScan LIZ-600 size standard (Applied Biosystems, Mulgrave, Victoria, Australia). The resulting electrophoretograms were analyzed using VivaxGEN version 1.0 and verified manually. ${ }^{23}$ To reduce potential artifacts from background noise, an arbitrary fluorescent intensity threshold of 50 relative fluorescence units was used. Only samples with information in at least $50 \%$ of the loci were considered as successfully genotyped.

Data analysis. Population diversity and genetic differentiation. Diversity was estimated by means of allelic richness (Rs). Rs was calculated by quantifying the number of alleles and normalizing the value to the sample size, using the hierfstat package in $R^{24}$ The expected heterozygosity $\left(H_{\mathrm{E}}\right)$, which is defined as the probability of finding a different allele at a specific locus when randomly selecting a pair of samples from the same population, was measured as an additional index of population diversity. The pairwise $F_{\mathrm{ST}}$ metric was used to determine the genetic distance between populations and interpreted according to the classification of Balloux and Lugon-Moulin ${ }^{25}$ (i.e., low if the $F_{\mathrm{ST}}$ value was 
less than 0.05; moderate if between 0.05 and 0.15 ; high if between 0.15 and 0.25 ; and very high if greater than 0.25 ). Arlequin software (version 3.5) was used to calculate $H_{\mathrm{E}}, F_{\mathrm{ST}}$, and the standardized measure $\left(F_{\mathrm{ST}}\right)$, which adjusts for high marker diversity. Significance values for the $F_{\mathrm{ST}}$ were derived using 10,000 permutations of the data in Arlequin.

Linkage disequilibrium (LD). To assess changes in transmission over time and to further characterize the parasites constituting the different subgroups, multilocus LD was measured using the standardized index of association $\left(I_{A}{ }^{S}\right)$. First, multilocus genotypes (MLGs) were reconstructed from the predominant allele at each locus in isolates with no missing data. Then, using the MLGs, $I_{A}{ }^{s}$ was measured using the webbased LIAN 3.5 software, assessing the significance of the estimates using 10,000 permutations of the data. ${ }^{26}$ The analysis was also performed using low-complexity infections (maximum of one multiallelic locus) and with each unique MLG represented just once.

Polyclonality and multiplicity of infection. Malaria parasites are haploid during the asexual stage; hence, the presence of more than one allele at a given locus in an individual sample is indicative of multiple parasite clones. In contrast to single nucleotide polymorphism-based markers, which would only reach up to four variants, the abundance of alleles at STR markers greatly facilitates the detection of polyclonal infections. However, the quality and quantity of parasite DNA may impact the sensitivity to detect minor alleles in polyclonal infections. In theory, minor clones are more likely to be identified in samples with larger quantities of parasite DNA. For this reason, Anderson and colleagues introduced the application of a minimum relative peak intensity threshold for calling minor alleles to enhance the comparability of multiplicity of infection (MOI) and polyclonality estimates between parasite populations with differing DNA quality or quantities. ${ }^{20}$ In view of the different sample qualities and quantities evaluated in the current study, minor alleles were only called if their height was at least 33\% of the major allele's height: a threshold that has been applied in multiple previous population genetic studies of malaria. ${ }^{27}$ An infection was defined as polyclonal if more than one allele was observed at any locus. The $\mathrm{MOI}$ for a given sample was defined as the maximum number of alleles at any locus. The number of polyclonal loci per sample was also estimated for closer inspection of the complexity of individual infections in the effort to identify any subtle differences between groups.

Statistical tests. R and SPSS software were used for statistical analysis with a significance level at $P<0.05 .^{28}$ Subgroup analysis was performed according to 1) patient recruitment method (active or passive) and 2) infection patency (i.e., patent or subpatent) using the $x^{2}$ test and Fisher's Exact Test. Differences in $\mathrm{MOI}$ and $H_{\mathrm{E}}$ between subgroups and species were assessed using the Mann-Whitney $U$ or Kruskal-Wallis test.

Ethics approval. Ethical approval for this study has been obtained from the Eijkman Institute Research Ethics Commission, Eijkman Institute for Molecular Biology, Jakarta, Indonesia (EIREC-47, EIREC-67, and EIREC-75), and the Human Research Ethics Committee of the Northern Territory Department of Health \& Families and Menzies School of Health Research, Darwin, Australia (HREC 2010-1396).

\section{RESULTS}

Marker properties. A summary of the diversity and genotyping success rate for each of the $P$. falciparum and $P$. vivax markers in all genotyped samples is presented in Supplemental Table 1. Moderate to high diversity was found for all $P$. falciparum markers except for TA42 and TA109, particularly in the TES and ESS samples (Supplemental Table 1). By contrast, high diversity was found in all $P$. vivax markers across all sample sets. However, the MS8 assay presented artifacts that affected the reliability of the allele calling and this locus was, therefore, excluded from further analysis. Genotype information for $50 \%$ of the marker set (five loci for $P$. falciparum and four loci for $P$. vivax) was obtained from $84.5 \%$ (414/492) $P$. falciparum samples and from $70.7 \%$ (390/552) of $P$. vivax samples. Most of the failed samples were asymptomatic $P$. vivax or $P$. falciparum infections (97\%; $157 / 162$ and $99 \%$; 77/78, respectively).

Sampling groups. Passively detected cases. All passively detected cases presented with microscopically confirmed parasitaemia. The comparability of the isolates collected from the clinical and ex vivo studies were assessed before pooling. No difference was observed between the median parasitaemia of the TES and ESS samples either in $P$. falciparum ( $N=45$, median TES $_{12,240}$ parasites $\mu L^{-1}$; and $N=94$, median $_{\mathrm{ESS}} 37,143$ parasites $\left.\mu \mathrm{L}^{-1} ; P=0.145\right)$ or $P$. vivax $\left(N=46\right.$, median TES $_{11,041}$ parasites $\mu \mathrm{L}^{-1}$; and $N=104$, median $_{\mathrm{ESS}} 16,224$ parasites $\mu \mathrm{L}^{-1} ; P=0.306$ ). Furthermore, given that the TES and ESS samples were collected across multiple years, an assessment of temporal differences in $\mathrm{MOI}$, polyclonality, $\mathrm{LD}, H_{\mathrm{E}}$, and $R s$ was undertaken with grouping by year of collection. Over the 6 years of sample collection, the proportion of polyclonal infections for $P$. falciparum varied from $0 \%$ to $31 \%,(P=0.085)$ and from $23 \%$ to $67 \%(P=0.104)$ for $P$. vivax, whilst the mean $\mathrm{MOI}$ ranged from 1.0 to 1.3 for $P$. falciparum, and from 1.2 to 2.0 for $P$. vivax $(P=0.085$ and $P=0.104$, respectively; Supplemental Table 2). Population diversity $\left(H_{\mathrm{E}}\right)$ values ranged from 0.448 to 0.608 for $P$. falciparum $(P=0.648$ ) and from 0.840 to 0.881 for $P$. vivax $(P=0.932)$. Furthermore, there was no difference between $R s$ for $P$. falciparum $\left(R_{S}=2.571-3.328 ; P=0.333\right)$ or $P$. vivax $\left(R_{S}=4.927-5.481 ; P=0.429\right)$. LD demonstrated modest fluctuations between the study years, with a slight increase observed in the $P$. falciparum cases in 2015 (Supplemental Table 3). Nonetheless, because there were no significant differences in diversity or complexity of infection between the study years, all TES and ESS samples were pooled into a single group defined as the passively detected patent sample group (PP) for subsequent analyses.

Actively detected cases. Only 18 of the isolates collected by active case detection were symptomatic $(N=5 P$. vivax and $N=13 P$. falciparum). The small sample size did not allow comparisons with other subgroups and, therefore, these were excluded from further analyses. Because venous blood samples were only taken from adults, $\mathrm{MOI}$, polyclonality, $R s$, and $H_{\mathrm{E}}$ were compared between adults ( $\geq 15$ years) and children ( $<15$ years), but no significant differences were observed in any of these measures (Supplemental Table 4). Likewise, there were no significant differences in $\mathrm{MOI}$, polyclonality, $R s$, and $H_{\mathrm{E}}$ between isolates from capillary or venous samples (Supplemental Table 5). Samples were, therefore, pooled into the following two subgroups: actively detected patent 
infections (AP) and actively detected subpatent infections (AS). In summary, AP comprises asymptomatic cases with a slide positive for $P$. falciparum or $P$. vivax monoinfection, whereas AS comprises asymptomatic cases with a slide negative for malaria, but PCR positive for either $P$. falciparum or $P$. vivax monoinfection.

The demographic details of all subgroups are presented in Table 1. Similar characteristics were observed across the groups in all features other than parasitaemia. The overall median parasitaemia was similar between the two species (Mann-Whitney U Test, $P=0.482$ ), but significantly different between active and passively detected cases for both species $(P<0.0001)$.

Stage composition and within-host diversity. Isolates for ex vivo susceptibility testing (ESS) were only selected if the ring stage composition was $\geq 70 \%$ and thus, the potential confounding of parasite staging was assessed. After excluding the ESS and AS samples, $P$. falciparum infections almost all comprised predominantly ring stages in both actively detected patent (AP; 89.4\%, 84/94) and passively detected cases (PP, 97.8\%, 44/45; $P=0.104$ ). By contrast, the percentage of synchronous ring stage $P$. vivax infections was $11.1 \%(9 / 81)$ in AP compared with $41.3 \%(19 / 46)$ in PP samples $(P>0.001$; Supplemental Table 6).

Among the AP cases, $P$. falciparum infections showed similar complexity between synchronous $\left(\mathrm{MOI}_{\mathrm{S}}=1.23 ; \mathrm{SD} \pm\right.$ $0.4)$ and asynchronous infections $\left(\mathrm{MOl}_{\mathrm{A}}=1.30 ; \mathrm{SD} \pm 0.5\right.$; $P=0.604)$, with polyclonal infections present in $23 \%(19 / 84)$ and $30 \%(3 / 10)$ of the cases, respectively $(P=0.694)$. Similarly, $P$. vivax infections showed comparable complexity of infection between synchronous and asynchronous infections: $\mathrm{MOI}_{\mathrm{S}}=1.78(\mathrm{SD} \pm 1.1)$ versus $\mathrm{MOI}_{\mathrm{A}}=1.75(\mathrm{SD} \pm 0.8$; $P=0.820)$, as well as proportion of polyclonal infections: $44 \%$ (4/9) and 54\% (39/72), respectively $(P=0.728)$. Likewise, comparison between synchronous and asynchronous passively detected $P$. vivax cases (ESS and TES) revealed no significant difference in complexity of infection $\left(\mathrm{MOI}_{\mathrm{S}}=1.39\right.$ $( \pm 0.6)$ versus $\left.\mathrm{MOI}_{\mathrm{A}}=1.52( \pm 0.8) ; P=0.463\right)$, or percentage of polyclonal infections (34\% (42/123) versus $41 \%(11 / 27)$, $P=0.516$; Table 2). Because there was only one asynchronous $P$. falciparum infection among the passively detected cases, no further comparisons could be performed. However, when the three datasets were combined, the results remained similar (Table 2).

Parasite density and within-host diversity. Assessment of $\mathrm{MOI}$ and parasitaemia in patent infections revealed a weak, but significantly negative correlation between $\mathrm{MOI}$ and parasitaemia for $P$. vivax (rho $=-0.400, P=0.032$ ), but not for $P$. falciparum (rho $=-0.095, P=0.146$ ). However, the correlation was not apparent after excluding the ESS samples for neither $P$. vivax (rho $=-0.117, P=0.184$ ), nor $P$. falciparum (rho $=-0.133, P=0.112$ ).

Population diversity, population differentiation, and LD analysis. There was no significant difference in the $P$. falciparum $R$ s between AP and AS $(P=0.635)$, between AP and PP $(P=0.170)$, and between PP and AS $(P=0.095$; Table 3). Similarly, $P$. vivax $R$ s was comparable between AP and AS $(P=0.640)$, between AP and PP $(P=0.809)$, and between PP and AS ( $P=0.799$; Table 3$). H_{E}$ values were also comparable across the subgroups in both species (Table 3 ). $F_{\mathrm{ST}}$ values varied from 0.007 to 0.047 and -0.002 to 0.003 for $P$. falciparum and $P$. vivax, respectively. Likewise, no differentiation between subgroups was observed for $P$. falciparum or $P$. vivax infections (Table 4).

There was no evidence of significant LD in any of the $P$. vivax subgroups, with $I_{A}^{S}$ scores ranging from 0 to 0.008 (Table 5). By contrast, significant LD was observed in each of the $P$. falciparum subgroups, with higher $I_{\AA}^{S}$ scores, ranging from 0.05 to 0.15 (Table 5). The highest levels of LD were observed in the actively detected $P$. falciparum subgroups. Further analysis using unique MLGs did not reveal evidence of clonal expansion in any of the subgroups in either species (Table 5).

Within-host diversity: complexity of infection and polyclonality. As summarized in Table 3, the mean $\mathrm{MOI}$ and the proportion of polyclonal infections was similar between $\mathrm{AP}$ and $\mathrm{AS}$ infections in $P$. falciparum $\left(\mathrm{MOI}_{\mathrm{AP}}=1.2\right.$ $( \pm 0.42), 23 \%$; and $\mathrm{MOI}_{\mathrm{SP}}=1.2( \pm 0.39), 16 \% ; P=0.199$ and $P=$ 0.189 , respectively). Similar $\mathrm{MOI}$ and polyclonality rates were also observed between $\mathrm{AP}$ and $\mathrm{AS}$ infections in $P$. vivax $\left(\mathrm{MOI}_{\mathrm{AP}}=1.7[ \pm 0.84], 52 \% ;\right.$ and $\mathrm{MOI}_{\mathrm{AS}}=1.6[ \pm 0.69], 50 \% ; P=$ 0.407 and $P=0.721$, respectively).

Although actively detected $P$. falciparum cases showed a higher mean $\mathrm{MOI}$ and a higher proportion of polyclonal infections compared with the passively detected infections, the difference was only statistically significant between the

TABLE 1

Demographic data of all cases

\begin{tabular}{|c|c|c|c|c|c|c|}
\hline \multirow[b]{2}{*}{ Subgroups } & \multicolumn{3}{|c|}{ Age, ${ }^{\star} N(\%)$} & \multirow[b]{2}{*}{ Males $N(\%)$} & \multirow[b]{2}{*}{ Parasitaemia† median (range) } & \multirow[b]{2}{*}{ Total } \\
\hline & $<5$ & $5-15$ & $>15$ & & & \\
\hline \multicolumn{7}{|l|}{ P. falciparum ${ }^{13}$} \\
\hline Passively detected $\ddagger$ & 0 & $19(13.6)$ & $121(86.4)$ & $68(48.6)$ & $14,837(3,280-361,728)$ & 140 \\
\hline Actively detected patent§ & $9(8.6)$ & $31(29.5)$ & $65(61.9)$ & 49 (46.7) & $600(27-132,256)$ & 105 \\
\hline Actively detected subpatent $\|$ & $9(4.1)$ & $57(26.3)$ & $151(69.6)$ & $81(37.3)$ & $\mathrm{n} / \mathrm{a}$ & 217 \\
\hline All cases & $18(4.4)^{2}$ & $107(23.1)^{5}$ & $337(72.9)^{6}$ & $204(42.9)$ & $7,595(27-361,728)$ & 4,6213 \\
\hline \multicolumn{7}{|l|}{ P. vivax ${ }^{5}$} \\
\hline Passively detected $\neq$ & 0 & $28(18.2)$ & $126(81.8)$ & 75 (48.4) & $15,072(1,640-120,576)$ & 155 \\
\hline Actively detected patent§ & $20(23.3)$ & $25(29.1)$ & $41(47.7)$ & $40(46.5)$ & $385(38-19,200)$ & 86 \\
\hline Actively detected subpatent $\|$ & $17(7.4)$ & $72(31.4)$ & $140(61.1)$ & $90(39.3)$ & $\mathrm{n} / \mathrm{a}$ & 229 \\
\hline All cases & $37(8.0)^{1}$ & $125(26.4)$ & $311(65.6)^{4}$ & $207(43.6)$ & $7,884(38-120,576)$ & 4,755 \\
\hline
\end{tabular}

Superscript numbers indicate excluded symptomatic community survey samples.

${ }^{\star}$ Age in years.

$\dagger$ Parasites $/ \mu \mathrm{L}$

$\ddagger$ Symptomatic patent infections.

$\S$ Asymptomatic patent infections.

\| Asymptomatic subpatent infections. 
TABLE 2

Within-host diversity and ring stage synchronicity in Plasmodium falciparum and Plasmodium vivax sample sets

\begin{tabular}{|c|c|c|c|c|c|c|}
\hline Sample sets & Synchronous* & $N(\%)$ & MOI Mean (SD) $\dagger$ & $P \ddagger$ & Polyclonality $N(\%)$ & $P \S$ \\
\hline \multicolumn{7}{|l|}{ P. falciparum } \\
\hline \multirow[t]{2}{*}{ Actively detected cases $\|^{1}$} & Yes & $84(89)$ & $1.23(0.42)$ & 0.604 & $19(23)$ & 0.694 \\
\hline & No & $10(11)$ & $1.30(0.48)$ & & $3(30)$ & \\
\hline \multirow[t]{2}{*}{ Passively detected cases $₫$} & Yes & $138(99)$ & $1.12(0.33)$ & - & 17 (12) & - \\
\hline & No & $1(1)$ & - & & - & \\
\hline \multicolumn{7}{|l|}{ P. vivax } \\
\hline \multirow[t]{2}{*}{ Actively detected cases $\|^{6}$} & Yes & $9(11)$ & $1.78(1.09)$ & 0.820 & $4(44)$ & $0.728 \#$ \\
\hline & No & $72(89)$ & $1.75(0.82)$ & & 39 (54) & \\
\hline \multirow[t]{2}{*}{ Passively detected cases $\Upsilon^{5}$} & Yes & $123(82)$ & $1.39(0.60)$ & 0.463 & $42(34)$ & 0.516 \\
\hline & No & 27 (18) & $1.52(0.75)$ & & $11(41)$ & \\
\hline
\end{tabular}

$\mathrm{MOI}=$ multiplicity of infection

${ }^{\star}$ Ring stage $>70 \%$.

† Standard deviation.

$\ddagger$ Mann-Whitney $U$ test.

$\S x^{2}$.

C Community survey.

qTreatment efficacy study and ex vivo surveillance study.

\# Fisher's exact test.

\# Fisher's exact test.
${ }_{\text {** }}$ Superscript indicates number of cases with missing data.

proportion of polyclonal infections in the AP (23\%) compared with the PP cases (12\%; $P=0.035$; Table 3$)$. By contrast, when comparing $P$. vivax subgroups, the actively detected cases showed consistently higher mean $\mathrm{MOI}\left(\mathrm{MOI}_{\mathrm{AP}}=1.7,[ \pm 0.84]\right.$, $\left.\mathrm{MOI}_{A S}=1.6,[ \pm 0.69]\right)$ compared with the passively detected infections $\left(\mathrm{MOI}_{\mathrm{PP}}=1.4,[\mathrm{SD} \pm 0.63], P=0.003\right)$, and a higher proportion of polyclonal infections $(51 \%$ and $35 \%$, respectively, $P=0.003$; Table 3 ).

The number of multiallelic loci (MLOCI) in the polyclonal infections in each of the $P$. vivax and $P$. falciparum subgroups is illustrated in Figure 1. The actively detected polyclonal $P$. vivax infections presented a higher average $\mathrm{MLOCl}$ than those detected passively $\left(\mathrm{MLOCl}_{\mathrm{A}}=2.8,[ \pm 1.7], \mathrm{MLOCl}_{\mathrm{P}}=2.1,[ \pm 1.5]\right.$; $P=0.010$ ). The actively detected $P$. falciparum infections presented a lower average $\mathrm{MLOCl}$ than passively detected infections but the results were not statistically significant $\left(\mathrm{MLOCl}_{\mathrm{A}}=\right.$ 1.6, $\left.[ \pm 0.9], \mathrm{MLOCl}_{\mathrm{P}}=2.0,[ \pm 1.1] ; P=0.140\right)$.

\section{DISCUSSION}

This study presents a comprehensive comparative genetic analysis of actively versus passively detected cases of $P$. falciparum and $P$. vivax. Knowledge regarding potential sampling bias is vital for the effective design and interpretation of surveillance studies of malaria, particularly passive sampling strategies. The current study highlights the challenges that are associated with the interpretation of molecular data generated from different sampling sources and the key issues to consider for sampling strategies.

In a previous study conducted in Mimika district, genetic substructure was observed in the $P$. falciparum population, with a small subpopulation of infections found in actively detected cases only. ${ }^{15}$ On further investigation of the genetic differences between Plasmodium populations collected by active versus passive sampling strategies conducted in the same population, the current study revealed that there was no evidence of any significant differences in genetic diversity between the passively and actively detected subgroups in either $P$. falciparum or $P$. vivax. Furthermore, low differentiation between the subgroups in both $P$. falciparum and $P$. vivax confirmed that the different subgroups appear to comprise the same general pool of parasite strains. The highest levels of genetic differentiation were observed in comparisons of actively versus passively detected cases in $P$. falciparum $\left(F_{\mathrm{ST}}=\right.$ $0.023-0.05$; $F_{\text {ST }}^{\prime}=0.05-0.12$ ), likely reflecting the aforementioned small subpopulation of asymptomatic infections and possibly also inflated by modest temporal changes in LD. Indeed, LD analysis revealed higher allelic association among the actively detected $P$. falciparum cases in comparison to the passively detected cases. Nonetheless, the $F_{\mathrm{ST}}$ and $F_{\text {'ST }}$ values observed between the $P$. falciparum subgroups were all markedly lower than those reported by Noviyanti et al. ${ }^{29}$

TABLE 3

Within host and genetic diversity analysis of Plasmodium falciparum and Plasmodium vivax subgroups

\begin{tabular}{lccrr}
\hline \multicolumn{1}{c}{ Subgroups } & $N$ & MOI Mean ${ }^{*}( \pm \mathrm{SD})$ & Polyclonal infections $N(\%)$ & $R_{S} \dagger \mathrm{Mean}( \pm \mathrm{SD})$ \\
\hline P. falciparum & & & & \\
Passively detected§ & 139 & $1.1(0.329)$ & $(12)$ & $23(23)$ \\
Actively detected patent $\|$ & 100 & $1.2(0.423)$ & $27(16)$ & $5.771(2.81)$ \\
Actively detected subpatent & 164 & $1.2(0.393)$ & $50(19)$ & $6.476(2.06)$ \\
All actively detected & 264 & $1.2(0.405)$ & & $6.392(1.95)$ \\
P. vivax & & & $53(35)$ & \\
Passively detected & 150 & $1.4(0.626)$ & $43(52)$ & $78(50)$ \\
Actively detected patent & 82 & $1.7(0.843)$ & $121(51)$ & $17.790(7.20)$ \\
Actively detected subpatent & 155 & $1.6(0.686)$ & & $14.645(7.72)$ \\
All actively detected & 237 & $1.7(0.745)$ & & $16.540(8.80)$ \\
\hline
\end{tabular}

$\mathrm{MOI}=$ multiplicity of infection .

*Standard deviation.

†Allelic richness.

‡Symptomatic patent infections

§ Asymptomatic patent infections.

|| Asymptomatic subpatent infections. 
TABLE 4

Pairwise differentiation between Plasmodium falciparum and Plasmodium vivax subgroups

\begin{tabular}{|c|c|c|c|}
\hline P. falciparum & Passively detected $^{\star}$ & Actively detected patent† & Actively detected subpatentł \\
\hline Passively detected ${ }^{\star}$ & * & 0.0532 & 0.1191 \\
\hline Actively detected patent $†$ & $0.02287^{\star \star}$ & * & 0.021 \\
\hline Actively detected subpatent $\ddagger$ & $0.04713^{\star \star \star}$ & $0.00707^{\mathrm{NS}}$ & * \\
\hline \multicolumn{4}{|l|}{ P. vivax } \\
\hline Passively detected ${ }^{*}$ & * & 0.0131 & 0.0205 \\
\hline Actively detected patent $†$ & $0.00194^{\mathrm{NS}}$ & * & -0.0100 \\
\hline Actively detected subpatentł & $0.00318^{N S}$ & $-0.00154^{\mathrm{NS}}$ & * \\
\hline
\end{tabular}

between different Indonesian islands $\left(F_{\mathrm{ST}}=0.08-0.41\right.$; $F_{\text {ST }}^{\prime}=0.23-0.78$.

The equivalence in population diversity and low differentiation between the ESS and the other sample subgroups suggests that despite the application of strict sample selection, ${ }^{10,11}$ the results of drug susceptibility testing are likely to be representative of the parasites circulating in the broader population. Therefore, if parasites with resistanceconferring mutations were arising in the community, they would be likely to be detected in the ex vivo surveillance study.

The results of the study have important implications for our understanding of the molecular epidemiology of the parasites beneath the surface of the "malaria-iceberg." This is especially relevant in the context of mass drug administration campaigns, as one of the greatest concerns of this practice is the selection of resistant parasites circulating in the community at very low frequencies. ${ }^{30}$ Our results suggest that passive surveillance studies are a reliable "index" of the parasite strains circulating in the broader population. However, direct comparison of known resistance genotypes in patent versus subpatent infections will be required to confirm these findings. ${ }^{31}$

Our study also suggests that parasites derived from passively detected symptomatic infections had similar genotypes to actively detected asymptomatic infections. These findings suggest that the development of clinical symptoms may be more attributable to host factors such as immunity and RBC polymorphisms, than to intrinsic parasite factors resulting in a higher multiplication rate. Similar results were reported by Ferreira et al. ${ }^{32}$ who explored the genetic background of $P$. falciparum parasites in cases of uncomplicated versus cerebral malaria, finding no evidence of closer relatedness (i.e., no evidence of strain specificity) among the cerebral cases. In a genetic study of $P$. falciparum and $P$. vivax in Colombia, Pacheco et al. ${ }^{33}$ also found no evidence of differences in the circulating genotypes between severe and uncomplicated cases in either species. However, further studies need to be performed to support this hypothesis.

These observations are particularly relevant when considering the sampling strategy for population genetic studies of $P$. falciparum and $P$. vivax. The similar estimates of diversity and low differentiation in comparisons of different blood withdrawal methods (capillary versus venous samples), disease presentation (symptomatic/passively detected versus asymptomatic/actively detected), and concentration of DNA (patent versus subpatent) in both species, indicate that diversity estimates are not affected by any of these conditions. Therefore, population diversity and other population genetic measures derived from predominant allele calls can be reliably estimated on pooled samples and effectively compared between different sample types.

Population genetic studies routinely use estimates of complexity of infection as indicators of transmission intensity. ${ }^{34}$ In contrast to the population-level estimates of diversity, which are based on frequencies of the major alleles in a given infection, assessments of the deeper complexity within infections revealed several differences between sampling groups. Actively detected cases exhibited a higher $\mathrm{MOI}$ than passively detected cases of both species, albeit only reaching statistical significance in $P$. vivax, but not in $P$. falciparum. This finding has important implications for sampling strategies in population genetic studies of $P$. vivax investigating within-host diversity.

TABLE 5

Linkage disequilibrium between actively detected patent infections (AP), actively detected subpatent infections (AS), and passively detected patent infections (PP)

\begin{tabular}{|c|c|c|c|c|c|c|}
\hline & & & Low complexity & & & \\
\hline Subgroup & All infections $\mathrm{N}$ & $I_{A}^{S}$ & N & $I_{A}^{S}$ & Unique MLGs N & $I_{A}^{S}$ \\
\hline \multicolumn{7}{|l|}{ P. vivax } \\
\hline Passively detected ${ }^{*}$ & 150 & $0.0062^{N S}$ & 123 & $0.0043^{\mathrm{NS}}$ & 119 & $0.0038^{N S}$ \\
\hline Actively detected patent $\dagger$ & 82 & $0.008^{\mathrm{NS}}$ & 49 & $-0.0001^{\mathrm{NS}}$ & 73 & $0.008^{\mathrm{NS}}$ \\
\hline Actively detected subpatentł & 155 & $-0.0067^{N S}$ & 107 & $-0.01^{\mathrm{NS}}$ & 67 & $-0.0067^{\mathrm{NS}}$ \\
\hline \multicolumn{7}{|l|}{ P. falciparum } \\
\hline Passively detected* & 139 & $0.0542^{\star \star \star}$ & 129 & $0.0543^{\star \star \star}$ & 95 & $0.02^{\star \star \star}$ \\
\hline Actively detected patent $\dagger$ & 100 & $0.1354^{\star \star *}$ & 158 & $0.1325^{\star \star \star}$ & 62 & $0.12^{\star \star \star}$ \\
\hline Actively detected subpatentł & 164 & $0.1462^{\star \star \star}$ & 85 & $0.146^{\star \star \star}$ & 71 & $0.13^{\star \star \star}$ \\
\hline
\end{tabular}

Only samples with no missing data were included in the analyses. ${ }^{\star \star \star} P<0.001$; NS $=$ not significant.

* Symptomatic patent infections.

† Asymptomatic patent infections.

$\ddagger$ Asymptomatic subpatent infections. 


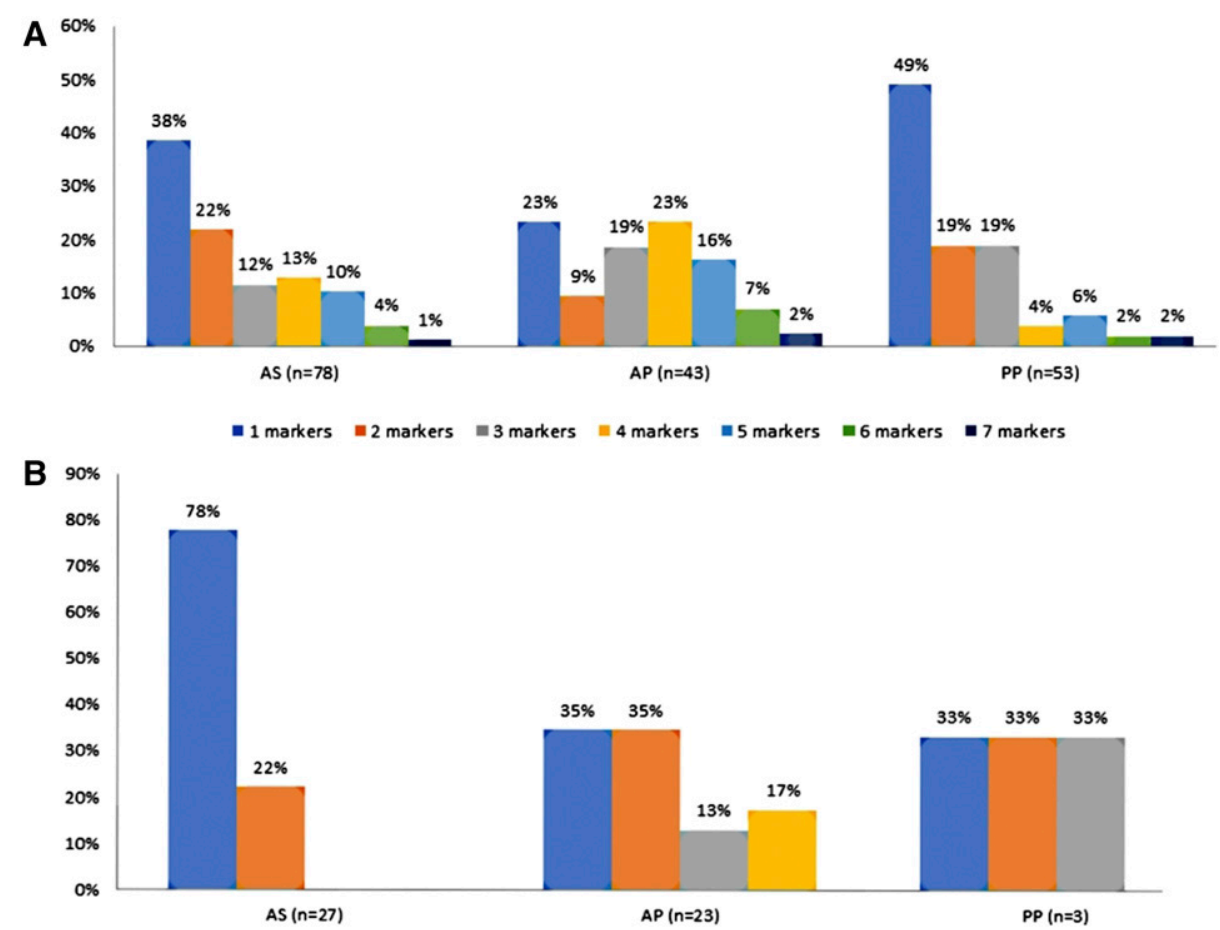

FIGURE 1. Polyclonal loci between asymptomatic subpatent infections (AS), asymptomatic patent infections (AP), and symptomatic patent infections (PP) in (A) Plasmodium vivax and (B) Plasmodium falciparum. This figure appears in color at www.ajtmh.org.

The contrast between the species could reflect interspecies differences in the persistence of subpatent infections resulting from new infections or relapses. In a recent longitudinal survey of individuals with asymptomatic malaria parasitaemia in a low endemic setting in Cambodia where $P$. vivax predominates, Tripura et al. $^{35}$ showed that $35 \%$ of individuals with $P$. vivax infection remained parasitaemic for up to 11 months, whereas only $13 \%$ of individuals with $P$. falciparum infection remained parasitaemic for up to 4 months. The persistence of asymptomatic parasitaemia might enhance the opportunity for multiple inoculations of $P$. vivax by different mosquitoes before the onset of clinical symptoms or parasite clearance.

Several previous studies have highlighted the challenges of characterizing multiple-clone infections using STR typing. ${ }^{27,36}$ These challenges mainly relate to the presence of stutter peaks or nonspecific PCR products that can interfere with the discrimination of artifacts from true minor alleles. Moreover, higher DNA concentrations can increase the detection of minor alleles. ${ }^{20,36,37}$ To counter potential differences in sample quality or quantity in population genetic studies of Plasmodium, minimum threshold values of $25 \%$ or $33 \%$ for calling additional alleles relative to the major allele are commonly applied. ${ }^{20,27,36}$ The negative correlation between parasitaemia and $\mathrm{MOI}$ in the current study suggests that applying a relative threshold such as $33 \%$ is not as effective in $P$. vivax samples as it was in $P$. falciparum samples when pooling samples from the community with those attending health facilities. Consequently, these results suggest caution when inferring transmission intensity from $\mathrm{MOI}$ and percentage of polyclonal infections from pooled samples.

The results of our study differ from those reported by Searle et al. ${ }^{14}$ who found that actively detected $P$. falciparum infections collected in a low endemic setting in Zambia were significantly divergent from passively detected cases, not only in terms of complexity of infection, but also in their genetic background. Key differences between the two studies that may explain the observed discrepancy are the greater temporal heterogeneity in sampling of active versus passively detected cases combined with greater bottlenecking in the $P$. falciparum population in the Zambian study. The Searle et al. $^{14}$ study shows evidence of increasingly unstable $P$. falciparum transmission over time, including increased inbreeding, consistent with rapidly declining transmission. These temporal genetic changes could account for the differences found between the beginning of the sampling period (when most of the actively detected cases were enrolled) and the end of the sampling period (when most of the passively detected cases were enrolled) as observed in other temporal genetic studies of $P$. falciparum. ${ }^{38,39}$ By contrast, the current study comprises samples collected in a period during which there was no marked change in malaria transmission and, with the actively detected cases enrolled within the sampling period of the passively detected cases. Differing from the situation in $P$. falciparum, reports on $P$. vivax population diversity remaining relatively resilient to changes in the intensity of transmission suggest that, ${ }^{12,34}$ except in areas with extreme bottlenecking such as Malaysia, ${ }^{40}$ passively detected cases should remain representative of the broader $P$. vivax population.

A limitation of this study is the large proportion of adult males missed from sampling during the cross-sectional study, which constitutes a bias per se that needs to be considered. The study also presents a potential bias reflecting the omission of very low-density infections that exhibited low genotyping success. Furthermore, the recently described high-volume, ultrasensitive PCR approach by Imwong et al. ${ }^{3}$ may have identified parasites at an even lower level of the "malaria-iceberg" that were not identified with the 
conventional PCR approach used here. However, it is also highly likely that these very low-density infections would be refractory to STR genotyping. ${ }^{3}$ Lastly, given the multiple factors affecting the dynamics of asymptomatic malaria and the influence of malaria transmission on parasite diversity and parasite differentiation, the results of the current study may not necessarily be extrapolated to areas with different malaria epidemiology. ${ }^{41,42}$ Indeed, available evidence inferred that the minor subpopulation of asymptomatic $P$. falciparum infections observed in Mimika district might reflect infections imported from other parts of Indonesia. ${ }^{16}$ As local transmission declines, the impact of imported cases on the parasite population diversity and structure may become more pronounced. ${ }^{40}$

In conclusion, asymptomatic malaria infections constitute a major challenge in pre-elimination areas where their prevalence may force the need to modify ongoing case detection strategies for surveillance. Many studies continue to use passively detected samples as an index of diversity and drug resistance in the broader parasite population. Our results suggest that passively detected samples provide effective representation of the 'strains' circulating in the broader community including the hidden asymptomatic reservoir. However, passive sampling strategies may not be effective for investigation of within-host infection complexity in the asymptomatic reservoir. These results highlight critical considerations when undertaking molecular surveillance of malaria.

Received May 8, 2017. Accepted for publication July 16, 2017.

Published online September 5, 2017.

Note: Supplemental tables appear at www.ajtmh.org.

Acknowledgments: We would like to thank the study participants and their parents or legal guardians who made themselves available for both the surveillance study at the hospital and the community survey in 2013. We are also grateful to Lembaga Pengembangan Masyarakat Amungme Kamoro and the staff of the RSMM hospital for their hard work and support for this study. Furthermore, we would like to thank all field and laboratory staff members of the Research Facility at the Papuan Health and Community Development Foundation (PHCDF) in Timika, Papua, Indonesia, for their efforts and support.

Financial support: The study was supported by the Wellcome Trust (a Senior Fellowship in Clinical Science awarded to RNP - 200909) and Fellowships to: JRP (Wellcome Trust - 099875), ZP (Instituto Colombiano para el Desarrollo de la Ciencia y la Tecnología Francisco José de Caldas, Colciencias - 512-2010) and NMA (NHMRC). The Timika Research Facility and Papuan Health and Community Development Foundation were supported by DFAT (Australian Department of Foreign Affairs and Trade) and the NHMRC (Program Grant 1037304).

Authors' addresses: Zuleima Pava, Irene Handayuni, Grennady Wirjanata, Steven Kho, Nicholas Anstey, Jutta Marfurt, and Sarah Auburn, Global and Tropical Health Division, Menzies School of Health Research, Charles Darwin University, Darwin, Northern Territory, Australia, E-mails: scribem13@gmail.com, fyireneh@ gmail.com, gwirjanata@gmail.com, steven.kho@menzies.edu.au, nicholas.anstey@menzies.edu.au, jutta.marfurt@menzies.edu.au, and sarah.auburn@menzies.edu.au. Leily Trianty, Retno A. S. Utami, Yusrifar K. Tirta, Agatha M. Puspitasari, and Rintis Noviyanti, Eijkman Institute of Molecular Biology, Jakarta, Indonesia, E-mails: leily@eijkman.go.id, tami@eijkman.go.id, r.yusrifar@gmail.com, agatha@ eijkman.go.id, and rintis@eijkman.go.id. Faustina Burdam and Jeanne Rini Poespoprodjo, Mimika District Health Authority, Timika, Papua, Indonesia, Timika Malaria Research Programme, Papuan Health and Community Development Foundation, Timika, Papua, Indonesia, and Child Health, Universitas Gadjah Mada, Yogyakarta, Indonesia, E-mails: lenny_burdam@yahoo.co.id and didot2266@yahoo.com. Enny Kenangalem, Mimika District Health Authority, Timika, Papua, Indonesia, and Timika Malaria Research Programme, Papuan Health and
Community Development Foundation, Timika, Papua, Indonesia, E-mail: ennykenangalem@yahoo.com. Hidayat Trimarsanto, Eijkman Institute of Molecular Biology, Bioinformatic Unit, Jakarta, Indonesia, and Badan Pengkajian da Penerapan Teknologi, Jakarta, Indonesia, E-mail: anto@eijkman.go.id. Ric N. Price, Global and Tropical Health Division, Menzies School of Health Research, Charles Darwin University, Darwin, Northern Territory, Australia, and Centre for Tropical Medicine, Nuffield Department of Clinical Medicine, University of Oxford, Oxford, Oxfordshire, United Kingdom, E-mail: ric.price@menzies.edu.au.

This is an open-access article distributed under the terms of the Creative Commons Attribution License, which permits unrestricted use, distribution, and reproduction in any medium, provided the original author and source are credited.

\section{REFERENCES}

1. Feachem RG, Sabot OJ, 2007. Global malaria control in the 21st century: a historic but fleeting opportunity. JAMA 297: 2281-2284.

2. WHO, 2016. Eliminating Malaria. Geneva, Switzerland: WHO, 24.

3. Imwong M, Hanchana S, Malleret B, Renia L, Day NP, Dondorp A, Nosten F, Snounou G, White NJ, 2014. High-throughput ultrasensitive molecular techniques for quantifying low-density malaria parasitemias. J Clin Microbiol 52: 3303-3309.

4. Phommasone $\mathrm{K}$ et al., 2016. Asymptomatic Plasmodium infections in 18 villages of southern Savannakhet Province, Lao PDR (Laos). Malar J 15: 296.

5. Waltmann A et al., 2015. High rates of asymptomatic, submicroscopic Plasmodium vivax infection and disappearing Plasmodium falciparum malaria in an area of low transmission in Solomon Islands. PLoS Negl Trop Dis 9: e0003758.

6. Britton S, Cheng Q, McCarthy JS, 2016. Novel molecular diagnostic tools for malaria elimination: a review of options from the point of view of high-throughput and applicability in resource limited settings. Malar J 15: 88.

7. Cheng Q, Cunningham J, Gatton ML, 2015. Systematic review of sub-microscopic $P$. vivax infections: prevalence and determining factors. PLoS Negl Trop Dis 9: e3413.

8. Bousema T, Okell L, Felger I, Drakeley C, 2014. Asymptomatic malaria infections: detectability, transmissibility and public health relevance. Nat Rev Microbiol 12: 833-840.

9. Wu L, van den Hoogen LL, Slater H, Walker PG, Ghani AC, Drakeley CJ, Okell LC, 2015. Comparison of diagnostics for the detection of asymptomatic Plasmodium falciparum infections to inform control and elimination strategies. Nature 528: S86-S93.

10. Russell B et al., 2008. Determinants of in vitro drug susceptibility testing of Plasmodium vivax. Antimicrob Agents Chemother 52: 1040-1045.

11. Kerlin DH, Boyce K, Marfurt J, Simpson JA, Kenangalem E, Cheng Q, Price RN, Gatton ML, 2012. An analytical method for assessing stage-specific drug activity in Plasmodium vivax malaria: implications for ex vivo drug susceptibility testing. PLoS Negl Trop Dis 6: e1772.

12. Barry AE, Waltmann A, Koepfli C, Barnadas C, Mueller I, 2015. Uncovering the transmission dynamics of Plasmodium vivax using population genetics. Pathog Glob Health 109: 142-152.

13. Arnot $D, 1998$. Unstable malaria in Sudan: the influence of the dry season. Clone multiplicity of Plasmodium falciparum infections in individuals exposed to variable levels of disease transmission. Trans $R$ Soc Trop Med Hyg 92: 580-585.

14. Searle KM et al.; Southern Africa International Centers of Excellence for Malaria Research, 2017. Distinct parasite populations infect individuals identified through passive and active case detection in a region of declining malaria transmission in southern Zambia. Malar J 16: 154.

15. Pava $Z$ et al., 2017. Genetic micro-epidemiology of malaria in Papua Indonesia: extensive $P$. vivax diversity and a distinct subpopulation of asymptomatic $P$. falciparum infections. PLoS One 12: e0177445.

16. Pava $Z$ et al., 2016. Submicroscopic and asymptomatic Plasmodium parasitaemia associated with significant risk of anaemia in Papua, Indonesia. PLoS One 11: e0165340.

17. Elyazar IR, Hay SI, Baird JK, 2011. Malaria distribution, prevalence, drug resistance and control in Indonesia. Adv Parasitol 74: 41-175. 
18. Auburn S et al., 2013. Effective preparation of Plasmodium vivax field isolates for high-throughput whole genome sequencing. PLoS One 8: e53160.

19. Singh B, Bobogare A, Cox-Singh J, Snounou G, Abdullah MS, Rahman HA, 1999. A genus- and species-specific nested polymerase chain reaction malaria detection assay for epidemiologic studies. Am J Trop Med Hyg 60: 687-692.

20. Anderson TJ, Su XZ, Bockarie M, Lagog M, Day KP, 1999. Twelve microsatellite markers for characterization of Plasmodium falciparum from finger-prick blood samples. Parasitology 119: 113-125.

21. Karunaweera ND, Ferreira MU, Munasinghe A, Barnwell JW, Collins WE, King CL, Kawamoto F, Hartl DL, Wirth DF, 2008. Extensive microsatellite diversity in the human malaria parasite Plasmodium vivax. Gene 410: 105-112.

22. Koepfli C, Mueller I, Marfurt J, Goroti M, Sie A, Oa O, Genton B, Beck HP, Felger I, 2009. Evaluation of Plasmodium vivax genotyping markers for molecular monitoring in clinical trials. $J$ Infect Dis 199: 1074-1080.

23. Trimarsanto H et al., 2017. VivaxGEN: an open access platform for comparative analysis of short tandem repeat genotyping data in Plasmodium vivax populations. PLoS Negl Trop Dis 11: e0005465.

24. Goudet J, 2005. HIERFSTAT, a package for $R$ to compute and test hierarchical F-statistics. Mol Ecol Notes 5: 184-186.

25. Balloux F, Lugon-Moulin N, 2002. The estimation of population differentiation with microsatellite markers. Mol Ecol 11: 155-165.

26. Haubold B, Hudson RR, 2000. LIAN 3.0: detecting linkage disequilibrium in multilocus data. Linkage analysis. Bioinformatics 16: 847-848.

27. Havryliuk T, Orjuela-Sanchez P, Ferreira MU, 2008. Plasmodium vivax: microsatellite analysis of multiple-clone infections. Exp Parasitol 120: 330-336.

28. Team RC, 2013. R: A Language and Environment for Statistical Computing. Vienna, Austria: R Foundation for Statistical Computing.

29. Noviyanti $R$ et al., 2015. Contrasting transmission dynamics of co-endemic Plasmodium vivax and $P$. falciparum: implications for malaria control and elimination. PLoS Negl Trop Dis 9: e0003739.

30. Poirot E, Skarbinski J, Sinclair D, Kachur SP, Slutsker L, Hwang J, 2013. Mass drug administration for malaria. Cochrane Database Syst Rev CD008846.
31. Marfurt J, Smith TA, Hastings IM, Muller I, Sie A, Oa O, Baisor M, Reeder JC, Beck HP, Genton B, 2010. Plasmodium falciparum resistance to anti-malarial drugs in Papua New Guinea: evaluation of a community-based approach for the molecular monitoring of resistance. Malar J 9: 8.

32. Ferreira MU, Nair S, Hyunh TV, Kawamoto F, Anderson TJ, 2002. Microsatellite characterization of Plasmodium falciparum from cerebral and uncomplicated malaria patients in southern Vietnam. J Clin Microbiol 40: 1854-1857.

33. Pacheco MA, Lopez-Perez M, Vallejo AF, Herrera S, Arevalo-Herrera M, Escalante AA, 2016. Multiplicity of infection and disease severity in Plasmodium vivax. PLoS Negl Trop Dis 10: e0004355.

34. Auburn S, Barry AE, 2017. Dissecting malaria biology and epidemiology using population genetics and genomics. Int $J$ Parasitol 47: 77-85.

35. Tripura $\mathrm{R}$ et al., 2016. Persistent Plasmodium falciparum and Plasmodium vivax infections in a western Cambodian population: implications for prevention, treatment and elimination strategies. Malar J 15: 181.

36. Havryliuk T, Ferreira MU, 2009. A closer look at multiple-clone Plasmodium vivax infections: detection methods, prevalence and consequences. Mem Inst Oswaldo Cruz 104: 67-73.

37. Vafa M, Troye-Blomberg M, Anchang J, Garcia A, Migot-Nabias F, 2008. Multiplicity of Plasmodium falciparum infection in asymptomatic children in Senegal: relation to transmission, age and erythrocyte variants. Malar J 7: 17.

38. Escalante AA et al., 2015. Malaria molecular epidemiology: lessons from the International Centers of Excellence for Malaria Research Network. Am J Trop Med Hyg 93: 79-86.

39. Nkhoma SC, Nair S, Al-Saai S, Ashley E, McGready R, Phyo AP, Nosten F, Anderson TJ, 2013. Population genetic correlates of declining transmission in a human pathogen. Mol Ecol 22: 273-285.

40. Abdullah NR et al., 2013. Plasmodium vivax population structure and transmission dynamics in Sabah Malaysia. PLoS One 8: e82553.

41. Galatas B, Bassat Q, Mayor A, 2016. Malaria parasites in the asymptomatic: looking for the hay in the haystack. Trends Parasitol 32: 296-308.

42. Gatei W et al., 2014. Genetic diversity of Plasmodium falciparum parasite by microsatellite markers after scale-up of insecticidetreated bed nets in western Kenya. Malar J 13 (Suppl 1): 495. 